\title{
Wavelet-domain Image Restoration Model Based on Variation Regularization
}

\author{
Caixia $\mathrm{Li}^{1}$, Chanjuan $\mathrm{Liu}^{2, *}$ and Hailin $\mathrm{Zou}^{3}$ \\ ${ }^{1}$ School of Information and Electrical Engineering, Ludong University \\ Yantai, China \\ 12000detian@163.com, ${ }^{2}$ luckycj80@sina.com, ${ }^{3}$ zhl_8655@sina.com
}

\begin{abstract}
There are two disadvantages in variational regularization based image restoration model. Firstly, the restored image is susceptible to noise because the diffusion coefficient depends on image gradient. Secondly, in the process of energy minimization, the selection of Lagrange multiplier $\lambda$ which is used to balance the regular term and the fidelity term can directly affects the quality of the restored image. To solve the above problems, the multi-resolution feature of multi-scale wavelet is introduced into the energy minimization model and a wavelet based image restoration model is proposed. In the proposed model, Lagrange multiplier $\lambda$ is replaced by an adaptive weighting function $\lambda_{j}$ which is constructed by the image wavelet transform coefficients. Theoretical analysis and experiment results show that, comparing with other methods, the proposed model reduces iterations in the energy minimization process, overcomes the cartoon effects in variational model and pseudo-Gibbs effect in traditional wavelet threshold methods, and can well protect the detail features while denoising.
\end{abstract}

Keyword: wavelet transform; total variation; energy minimization; image restoration

\section{Introduction}

Currently, the main methods for image denoising include stochastic modeling theory, wavelet analysis theory and partial differential equation(PDE) theory. PDE-based image denoising methods, according to the derivation of the PDE, can be divided into two categories, one is process-oriented methods which remove the noise according to the evolutionary theory in physics, mainly included diffusion model, P-M model [1], etc. The other is object-oriented methods, also called variational methods [2, 4, 7-9]. This kind of methods constructs an energy functional equation according to the inherent features of the original image. To achieve the purpose of image denoising, Rudin, Osher and Fatemi proposed a TV-based ROF model [2] which transformed an image denoising problem into a solution of function extreme value by introducing energy function. The above PDE-based methods are all based on the idea that image can be approximated by those piecewise smooth polynomials. But this representation has a poor ability in describing local time-frequency features, meanwhile, all of these methods use image gradient as the unique features quantity to describe image features, as we know, image gradient is susceptible to noise, thus it may be resulting in adverse visual effects for the restored image.

Using the local time-frequency features of wavelet transform can well distinguish the target signals from noises; therefore, wavelet transform based image denoising methods is just based on this principle. This kind of methods mainly includes wavelet threshold shrinkage denoising method [3], wavelet transform modulus maxima denoising method

${ }^{*}$ Corresponding Author 
and correlation denoising method. There are the three kinds of wavelet threshold shrinkage denoising methods. Thereinto, the hard threshold method can well preserve the image edges and other local features, but the improper threshold is likely to cause the loss of high frequency information and results in pseudo-Gibbs and other visual distortion. Compared with hard threshold methods, soft threshold methods can get smoother restored image on the vision, but it is easy to blur the image edges. The third method is semi-soft and semi-hard threshold method, but it is difficult to choose proper thresholds $T_{1}$ and

$T_{2}$ to achieve good compromise between hard threshold and soft threshold. Modulus maxima denoising method takes advantage of the difference singularity between signal and noise to observe the change rules of wavelet transform's modulus maxima under different scales. And then using alternating projection method, the image can be reconstructed by the reserved modulus maxima points. But alternately projection algorithm is complex, it is likely to cause deviation of the projection signal, thus it is difficult to process real-time signal in practical applications. Correlation denoising methods remove the noise according to the principle that the signal's wavelet coefficients after wavelet transform have strong correlation among each scale but the noise's wavelet coefficients have not such a clear correlation. But on the whole, the above image denoising methods based on wavelet transform are easily to cause edge distortion and position drift.

To overcome the above problems existing in PDE-based methods and wavelet transform methods. A new PDE-based image restoration model in wavelet domain is proposed which combines the good edge and shape preservation features in TV method with the wavelet transform method's local time-frequency characteristics. In the proposed model, in order to better balance the regular item and fidelity item, we take advantage of wavelet coefficients module value to construct an adaptive weighting function $\lambda_{j}$ which can better balance regular item and fidelity item under different scales and different sub-frequency.

\section{The Total Variation Regularization Model}

The classical variation denoising algorithm is the total variation denoising model proposed by Rudin,Osher and Fatemi, and it is also called ROF model[2]. This algorithm attempts to seek a minimal energy of energy functional.

Let the observed intensity function $u_{0}(x, y)$ denote the pixel values of a noisy image for $x, y \in \Omega$. Let $u(x, y)$ denote the desired clean image, so

$$
u_{0}(x, y)=u(x, y)+n(x, y)
$$

When $n$ is the additive noise.

An equation with energy minimal limiting condition is proposed as follow

$$
\min \quad E(u)=\iint_{\Omega}|\nabla u| d x d y
$$

where $u$ subjects to

$$
\left\{\begin{array}{c}
\iint_{\Omega} u(x, y) d x d y=\iint_{\Omega} u_{0}(x, y) d x d y \\
\frac{1}{|\Omega|} \iint_{\Omega}\left(u(x, y)-u_{0}(x, y)\right){ }^{2} d x d y=\sigma^{2}
\end{array}\right.
$$

When the noise is approximated by an additive white Gaussian process of standard deviation $\sigma$. Considering Lagrange multiplier $\lambda$, the problem can be translated into the energy minimization problem without limiting condition. 


$$
\min E(u)=\iint_{\Omega}|\nabla u| d x d y+\frac{\lambda}{2} \iint_{\Omega}\left|u(x, y)-u_{0}(x, y)\right|^{2} d x d y
$$

where the Lagrange multiplier is

$$
\lambda=\frac{1}{\sigma^{2}|\Omega|} \iint_{\Omega} \nabla \cdot \frac{\nabla u}{|\nabla u|}\left(u-u_{0}\right) d x d y
$$

The corresponding Euler-Lagrange Eq.(3) is:

$$
\nabla \cdot \frac{\nabla u}{|\nabla u|}+\lambda\left(u_{0}-u\right)=0
$$

From the above eq.(6), we know that the regular item is $1 /|\nabla u|$. As we know, when $|\nabla u|$ is large, it may be an edge or texture, or it may be a large noise point, so the denoising behavior should be weakened which will make the model has a certain boundary and texture protection ability, but it is also easy to confuse large noise with the edge and makes the noise be preserved; when $|\nabla u|$ is small, it means a smooth area of the image, so in these areas the model should have a strong denoising capability. Therefore, the TV model is an overall transform method, it can only describe the overall features of the image, so it can not reflect the image's time-frequency local features.

\section{Definition of Wavelet Transform Modulus and the Weighting Function}

Supposed that $\psi(x, y)$ is a 2-demension basic wavelet, for any $f(x, y) \in L^{2}\left(R^{2}\right)$, the continuous wavelet transform (CWT) can be defined as

$$
W_{a} f(x, y)=\iint_{R^{2}} f(u, v) \overline{\psi_{a, x, y}}(u, v) d u d v=<f(u, v), \psi_{a, x, y}(u, v)>,
$$

where $\varphi_{a, x, y}(u, v)=\varphi((u-x) / a,(v-y) / a) / a, a>0$ represents scale parameter. If $\varphi(x, y)$ is separable, then CWT has three components, that is level detail, vertical detail and diagonal detail. Here, we mainly use level detail and vertical detail to extract the edge information of images through wavelet transform. In the following discussion, we use $W_{a}^{k} f(x, y)=<f(u, v), \psi_{a, x, y}(u, v)>, k=1,2$ to express wavelet transform in this two directions, where $\varphi^{1}(x, y)$ and $\varphi^{2}(x, y)$ indicate the wavelet in this two directions separately. As we know, the square of wavelet transforms module is

$$
M=M_{a} f(x, y)=\left|W_{a}^{1} f(x, y)\right|^{2}+\left|W_{a}^{2} f(x, y)\right|^{2}
$$

Eq.(7) reflects the grey transformation in the point $f(x, y)$. Specifically, when in the smooth areas of image, $M_{a} f(x, y)$ is small; when in the edge, $M_{a} f(x, y)$ is large, and the singularity of the image gets stronger with the increase of $M_{a} f(x, y)$.Moreover, noise mainly focuses on small scale wavelet coefficients and its' wavelet coefficients decline rapidly with the increase of scale; image can be described by some large wavelet coefficients, and the influence of Gaussian white noise to each wavelet coefficient is small. Signal wavelet coefficient gets larger or stays unchanged with the increase of decomposition scale. Therefore, the wavelet transform coefficients can more accurately reflect the edge information. Here, using the wavelet modulus value to define the image structure measure is as follows: 


$$
\lambda_{\mathrm{j}}=\frac{N-\min (N)}{\max (N)-\min (N)} \quad N=\sqrt{M}
$$

In the TV model, in order to achieve the purpose of energy minimization, the Lagrange multiplier $\lambda$ is introduced to balance regular item and fidelity item. By the equation (5), the value of the Lagrange multiplier $\lambda$ is just dependent on the intensity of the noise and gradient value, which is susceptible to noise. And it has the same value in both the edge area and the smooth area, and has a poor adaptive ability. Now we use the image local adaptive information function $\lambda_{j}$ which is described by the wavelet transform modulus to instead of Lagrange multiplier $\lambda$ in TV model. Based on wavelet multi-resolution characteristics, $\lambda_{j}$ is selected depending on the signal and noise distribution under different scales to balance fidelity item and regular item, therefore, we can get better denoising and image feature protection effects.

\section{Wavelet Domain Image Variational Restoration Model}

Based on wavelet multi-scale features, a wavelet domain image restoration model based on variation regularization is proposed which can process TV model denoising on different scales. It compensates for the deficiencies of TV model that the overall TV transformation can not reflect time-frequency local features.

Noise model is given as follows:

$$
u_{0}(x)=u(x)+n(x)
$$

where $u(x)$ is the original image without noise, $n(x)$ is the image noise, $u_{0}(x)$ is a known noise image.

The standard wavelet transform of $u_{0}(x)$ is:

$$
u_{0}(x, \alpha)=\sum_{j \in I} \alpha_{j} \psi_{j}(x)
$$

where $\alpha=\left(\alpha_{j}\right)$ are wavelet coefficients, $\psi_{j}(x)$ is a wavelet function, $j$ represents wavelet scale, $u(x, \beta)$ is defined as:

$$
u(x, \beta)=\sum_{j \in I} \beta_{j} \psi_{j}(x)
$$

where $\beta=\left(\beta_{j}\right)$

Total variation model is defined as:

$$
F(u)=\int_{R^{2}}\left|\nabla_{x} u(x, \beta)\right| d x+\frac{1}{2} \sum_{j \in I} \lambda_{j}\left(\beta_{j}-\alpha_{j}\right)^{2}
$$

In eq.(13), Wavelet domain image restoration model based on variation regularization combines wavelet decomposition with variation theory. In the proposed model, we use the image wavelet modulus construct a weighting function to describe the image structure information in order to balance fidelity item and regular item.

Suppose that $u$ is a piecewise smooth function, then image denoising problem can be converted into solving an energy minimization problem.

$$
\min _{\beta} F(u)=\int_{R^{2}}\left|\nabla_{x} u(x, \beta)\right| d x+\frac{1}{2} \sum_{j \in I} \lambda_{j}\left(\beta_{j}-\alpha_{j}\right)^{2}
$$


Using the variational method to obtain the derivative of $F(u)$.

$$
\begin{aligned}
\frac{\partial F(u)}{\partial \beta_{j}}=\int_{R^{2}} \frac{\nabla_{x} u}{\left|\nabla_{x} u\right|} \cdot \nabla_{x} \psi \psi_{j} d x+\lambda_{j}\left(\beta_{j}-\alpha_{j}\right) \\
=-\int_{R^{2}} \nabla_{x} \cdot\left\lfloor\frac{\nabla_{x} u}{\left.\left|\nabla_{x} u\right|\right\rfloor} \mid \psi_{j} d x+\lambda_{j}\left(\beta_{j}-\alpha_{j}\right)\right.
\end{aligned}
$$

where $u=u(x, \beta)$, suppose wavelet function $\psi$ is compactly supported and satisfies Lipschitz continuous conditions, the corresponding Euler-Lagrange equation is

$$
-\int_{R^{2}} \nabla_{x} \cdot\left[\frac{\nabla_{x} u}{\left.\left|\nabla_{x} u\right|\right\rfloor} \mid \psi_{j} d x+\lambda_{j}\left(\beta_{j}-\alpha_{j}\right)=0\right.
$$

where $-\int_{R^{2}} \nabla_{x} \cdot\left[\frac{\nabla_{x} u}{\left|\nabla_{x} u\right|}\right] \psi_{j} d x=-\left\langle k_{k}, \psi_{j}\right\rangle$, eq.(16) well combines geometric information of the image(curvature $k$ ) with wavelet decomposition. After wavelet decomposition, we can respectively remove the noise according to the signal and noise distribution features under different resolutions. More precisely, $\beta=\beta(t)=\left(\beta_{j}(t)\right)$, so the time evolution equation is as following:

$$
\frac{\partial \beta_{j}}{\partial t}=\int_{R^{2}} \nabla_{x} \cdot\left[\frac{\nabla_{x} u}{\left|\nabla_{x} u\right|} \mid \psi_{j} d x-\lambda_{j}\left(\beta_{j}-\alpha_{j}\right) \quad \beta_{j}(0)=\alpha_{j}\right.
$$

Comparing eq. (5) with eq.(13), it shows that the image edges are well maintained in the diffusion process because the introduction of a significant second geometry information curvature in TV model. Wavelet domain image restoration model based on variation regularization combines curvature with wavelet decomposition. Based on the noise wavelet coefficients decreased fast with the scale increased, so that TV model combines with wavelet decomposition can choose different $\lambda_{j}$ according to different scales to keep better image detail features. And because the sparse distribution of wavelet coefficients makes iterations of WTV model quickly reduces.

\section{The Selection of Wavelet}

Because wavelet basis function has different categories and each category has different properties, so the selection of wavelet basis function depends on the following aspects:

1) orthogonality

Firstly, Orthogonality allows the L2 norm of the function $f$ to directly establish relation with the norm of its wavelet coefficients. Secondly, the fast wavelet transform is a unitary transform, and its inverse transform is easy to implement. Finally, it has the advantage of good computational stability, high precision and fine signal energy maintain ability.

\section{2) Symmetry}

If both the scaling function and wavelet function are symmetric, the filter will produce linear phase. Linear phase is not only suitable for human visual system, but also easy to handle the signal edge. 


\section{3) Compact Support}

Compact support is an important property of wavelet. The smaller wavelet support set is, the stronger its localization ability is, which makes it is more beneficial to determine the signal mutations. The correspondence filter of compactly supported wavelet is a finite impulse response(FIR) filter, so the sum of the corresponding fast wavelet transform is limited, while non-compactly supported wavelet must be truncated in the actual operation.

\section{4) Regularity}

Regularity is a description of the function smoothness degree, it is also a measure of energy concentration. The greater the regular order is, the smoother the function is, and which means more concentrated energy in the frequency domain and better processing effect. Meanwhile, regularity has a relationship with the size of the support set, the larger the support set is, the better the regularity is. Take the Daubechies wavelets and spline wavelets for example, smoothness increases with the increase of support set length, but the increase of support set length will extend the processing time.

\section{5) High Order Vanishing Moments}

Vanishing moment of the wavelet transform shows the degree of energy concentration. When the order of vanishing moments is large, under the fine-scale, some values of high frequency part are small enough to be negligible (except the singular point).

Table 1 shows several performances of common wavelet.

\section{Table 1. Several Common Wavelets Application Performance Analysis}

\begin{tabular}{|c|c|c|c|c|}
\hline $\begin{array}{l}\text { Wavelet } \\
\text { function }\end{array}$ & Haar & Daubechies & Biorthogonal & Symlets \\
\hline representation & haar & $\mathrm{dbN}$ & biorNr.Nd & symN \\
\hline orthogonality & has & has & no & Has \\
\hline biorthogonalit & has & has & has & has \\
\hline $\begin{array}{c}\mathrm{y} \\
\text { Compact } \\
\text { support }\end{array}$ & Has & has & Has & has \\
\hline $\begin{array}{c}\text { Length of } \\
\text { compact } \\
\text { support }\end{array}$ & 1 & $2 \mathrm{~N}-1$ & $\begin{array}{ll}\text { re: } & 2 \mathrm{Nr}+1 \\
\text { de: } & 2 \mathrm{Nd}+1\end{array}$ & $2 \mathrm{~N}-1$ \\
\hline Length of filter & 2 & $2 \mathrm{~N}$ & $\begin{array}{c}\operatorname{Max}(2 \mathrm{Nr}+2 \mathrm{Nd} \\
)+2\end{array}$ & $2 \mathrm{~N}$ \\
\hline symmetry & $\mathrm{S}$ & App S & AS & App S \\
\hline
\end{tabular}

We know that different images have different features, similarly, different wavelet bases also express different features when analyzing and reconstructing images. Essentially, the performances of wavelet based signal processing method firstly depend on inherent features of the signal; secondly, it depends on the selection of processing method. Once the signal to be processed has been selected, the most important thing is the choice of wavelet basis.

In order to verify different wavelet basis function on the result of image processing, we choose cartoon effect image Peppers, containing textures image Barb, medical image Brain and natural landscape image Goldhill, noise with $\sigma^{2}=20$ was added to these images, different wavelets were selected for experiment, Peak signal to noise (PSNR) and Mean absolute error (MAE) were as follows:

Table 2. Comparison Results of Selected Wavelet (PSNR)

\begin{tabular}{ccccc}
\hline & haar & Bior2.4 & Db4 & Sym4 \\
\hline peppers & 29.9610 & 29.7044 & 29.8331 & 29.9894 \\
barb & 28.7833 & 28.7082 & 28.8620 & 28.8428 \\
brain & 28.5693 & 28.5176 & 28.9880 & 29.0013 \\
goldhill & 28.5557 & 28.3603 & 28.5904 & 28.6067 \\
\hline
\end{tabular}


Table 3. Comparison Results of Selected Wavelet (MAE)

\begin{tabular}{ccccc}
\hline & haar & Bior 2.4 & Db4 & Sym4 \\
\hline peppers & 5.5903 & 5.8156 & 5.7405 & 5.6626 \\
barb & 6.6841 & 6.8141 & 6.7047 & 6.7120 \\
brain & 7.0627 & 7.1350 & 6.8866 & 6.7774 \\
goldhill & 7.0425 & 7.2849 & 7.0928 & 7.0277 \\
\hline
\end{tabular}

Table 2 shows that different wavelet bases have different processing results when processing the same image, and the same wavelet basis function has different processing effects when processing different images. Therefore, there is not a wavelet suitable for all types of image.

\section{Results and Analysis}

In order to verify the effectiveness of the method proposed in this paper(WTV), we performed sets of simulation experiments by MATLAB. Now using Peppers, Barb , Brain and Goldhill grayscale images as examples to explain and analyze, the size of images is $256 \times 256$ and grayscale is 256. First, adding different levels of zero-mean Gaussian noise to the original image as input. Taking Peak signal to noise (PSNR) and Mean absolute error (MAE) as objective criterions to evaluate the restored images treated by improved ROF TV model, wavelet thresholding model and the model proposed in this paper WTV.

Peak Signal to Noise Ratio(PSNR) is defined by:

$$
P S N R=10 \lg \frac{255^{2}}{\frac{1}{m \times n} \sum_{i=1}^{m} \sum_{j=1}^{n}\left(\tilde{u}_{i j}-u\right)^{2}}
$$

Mean absolute error (MAE) is defined by:

$$
M A E=\frac{1}{m \times n} \sum_{i=1}^{m} \sum_{j=1}^{n}\left|\tilde{u}_{i j}-u\right|
$$

where $\tilde{u}_{i j}$ is the gray value of the restored image and $u$ is the gray value of original image without pollution. $m \times n$ is the size of the image.

Figure 1 to Figure 9 show the image visual effect of images before and after noise reduction processing using three approaches separately(restored images by WTV model choose Db4 wavelet and Sym4 wavelet under 3 scale wavelet decomposition). Table4. shows the value of PSNR $(\mathrm{dB})$ using three different approaches under different noise levels. Table 5 shows the value of MAE using the three different approaches under different noise levels.

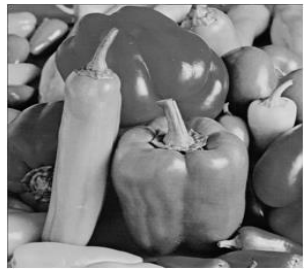

(a) Peppers

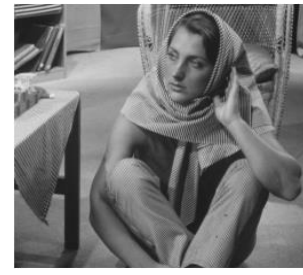

(b) Barb

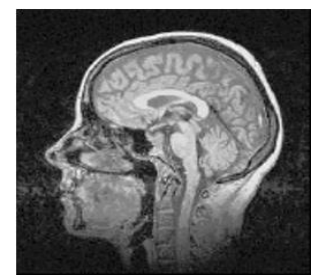

(c) Brain

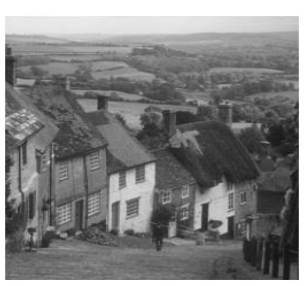

(d) Goldhill

Figure 1. Original Images 


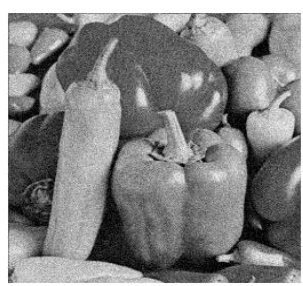

(a) Nosiy image

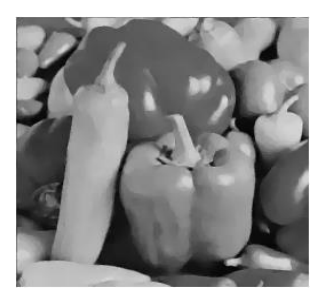

(b) ROF image

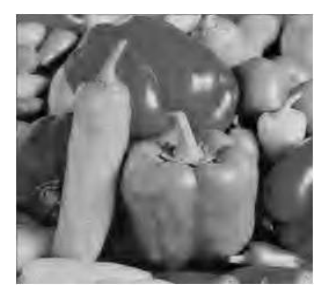

(c) $\mathrm{HT}$ image

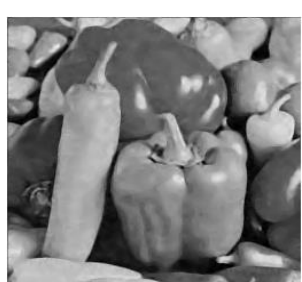

(d) WTV image

Figure 2. Noise Intensity $\sigma^{2}=15$, Denoising Effect of Peppers Image using Three Approaches

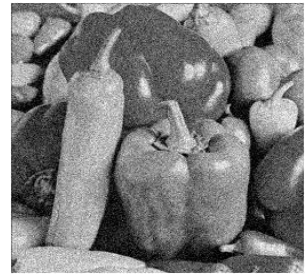

(a) Nosiy image

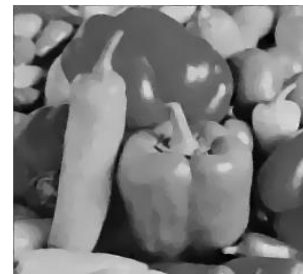

(b) ROF image

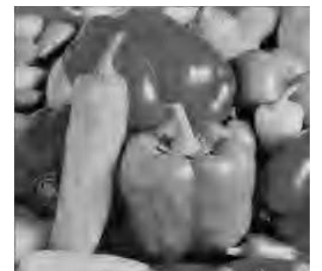

(c) HT image

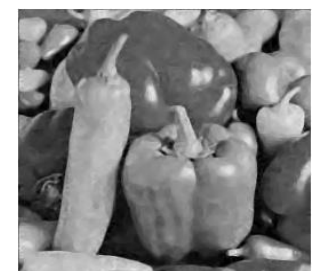

(d) WTV image

Figure 3. Noise Intensity $\sigma^{2}=20$, Denoising Effect of Peppers Image using Three Approaches

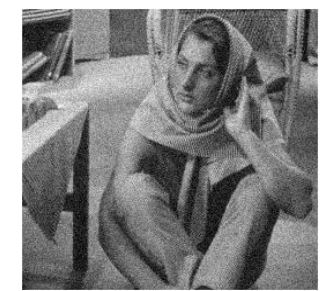

(a) Nosiy image

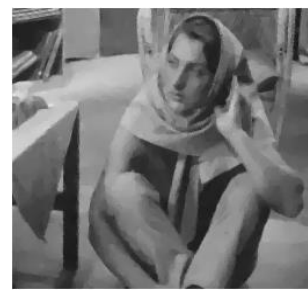

(b) ROF image

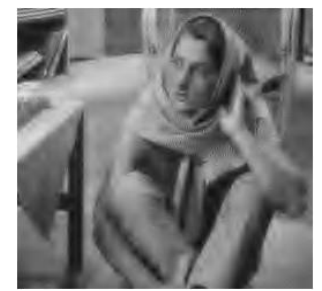

(c) $\mathrm{HT}$ image

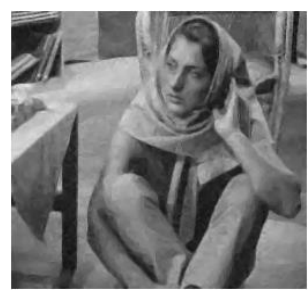

(d) WTV image

Figure 4. Noise Intensity $\sigma^{2}=15$, Denoising Effect of Barb Image using Three Approaches

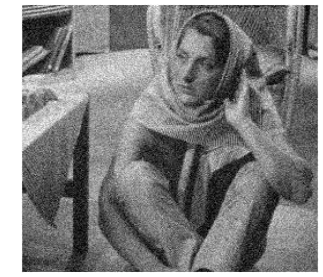

(a) Nosiy image

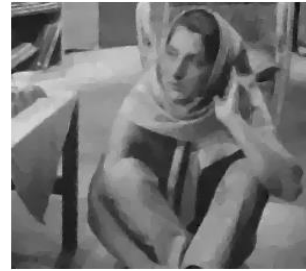

(b) ROF image

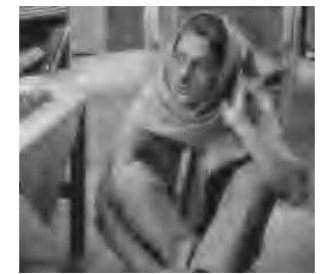

(c) $\mathrm{HT}$ image

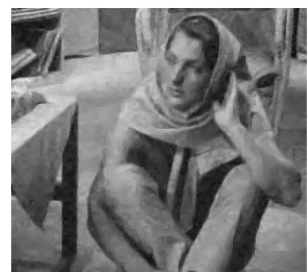

(d) WTV image

Figure 5. Noise Intensity $\sigma^{2}=20$, Denoising Effect of Barb Image using Three Approaches

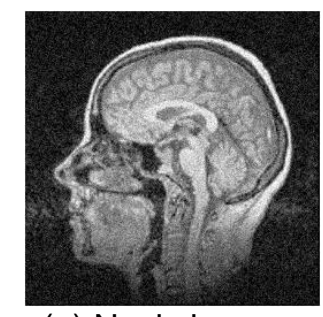

(a) Nosiy image

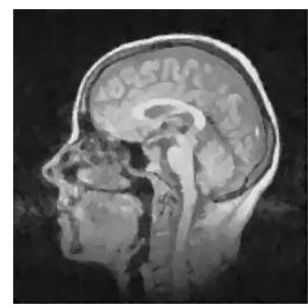

(b) ROF image

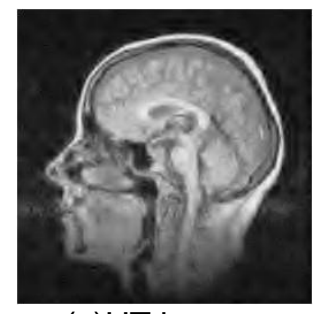

(c) $\mathrm{HT}$ image

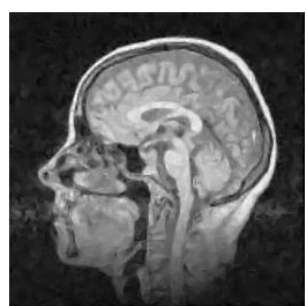

(d) WTV image

Figure 6. Noise Intensity $\sigma^{2}=15$, Denoising Effect of Brain Image using Three Approaches 


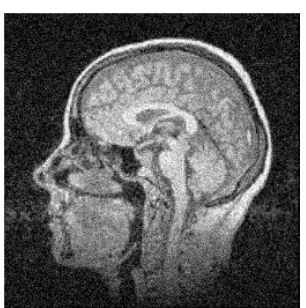

(a) Nosiy image

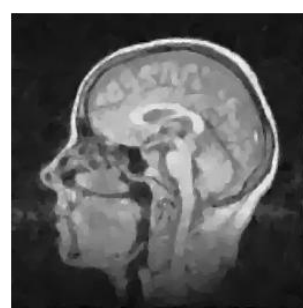

(b) ROF image

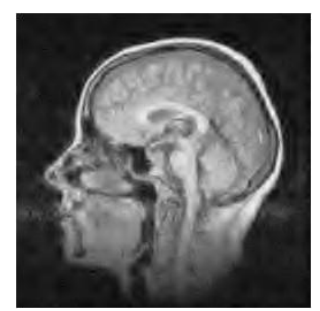

(c) HT image

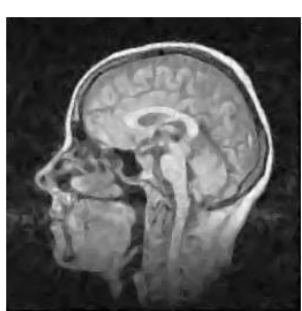

(d) WTV image

Figure 7. Noise Intensity $\sigma^{2}=20$, Denoising Effect of Brain Image using Three Approaches

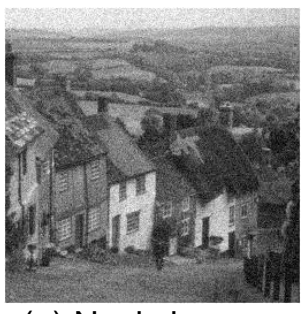

(a) Nosiy image

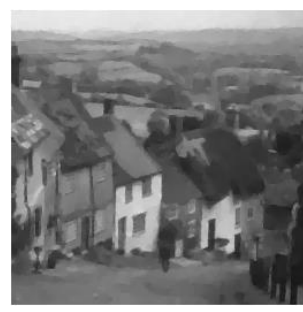

(b) ROF image

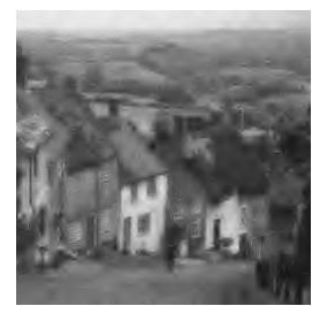

(c) $\mathrm{HT}$ image

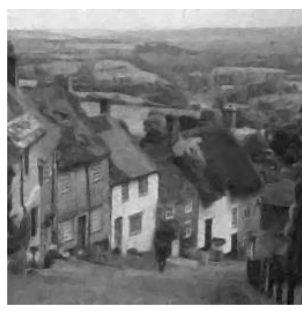

(d) WTV image

Figure 8. Noise Intensity $\sigma^{2}=15$, Denoising Effect of Goldhill Image using Three Approaches

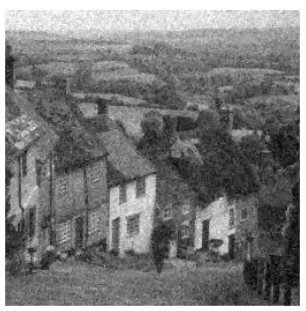

(a) Nosiy image

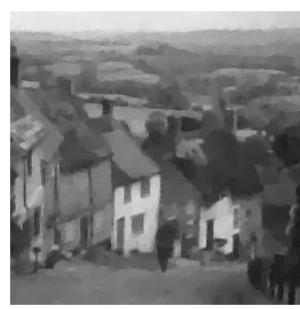

(b) ROF image

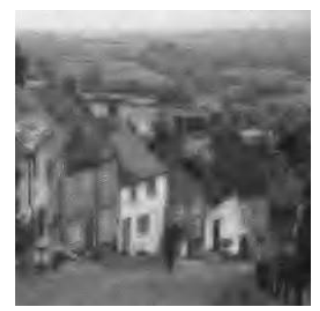

(c) $\mathrm{HT}$ image

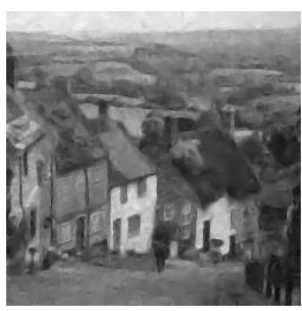

(d) WTV image

Figure 9. Noise Intensity $\sigma^{2}=20$, Denoising Effect of Goldhill Image using Three Approaches

Table 4. The Comparing Three Different Models of PSNR[dB]

\begin{tabular}{|c|c|c|c|c|c|c|c|c|}
\hline \multirow[b]{2}{*}{$\sigma^{2}$} & \multicolumn{4}{|c|}{ Peppers } & \multicolumn{4}{|c|}{ Barb } \\
\hline & $\begin{array}{c}\text { ROF } \\
\text { TV }\end{array}$ & Ying & $\mathrm{Db} 4$ & Sym4 & ROF TV & Ying & Db4 & Sym4 \\
\hline 10 & 33.0362 & 30.1715 & 33.0681 & 33.4314 & 31.2963 & 28.9488 & 31.2896 & 31.3895 \\
\hline 15 & 31.0193 & 28.0957 & 31.2271 & 31.3717 & 29.6489 & 27.7285 & 29.8108 & 29.8452 \\
\hline 20 & 29.6709 & 26.6577 & 29.8331 & 29.9894 & 28.5992 & 26.1676 & 28.8620 & 28.8428 \\
\hline \multirow[t]{2}{*}{25} & 28.6384 & 25.7140 & 28.7905 & 28.9220 & 27.7483 & 25.4294 & 27.7951 & 27.8289 \\
\hline & \multicolumn{4}{|c|}{ Brain } & \multicolumn{4}{|c|}{ Goldhill } \\
\hline$\sigma^{2}$ & $\begin{array}{c}\text { ROF } \\
\text { TV }\end{array}$ & Ying & $\mathrm{Db} 4$ & Sym4 & ROF TV & Ying & $\mathrm{Db} 4$ & Sym4 \\
\hline 10 & 31.5499 & 29.1170 & 31.4459 & 31.7108 & 31.4452 & 28.5223 & 31.5529 & 31.5214 \\
\hline 15 & 29.7502 & 27.2610 & 30.1584 & 30.3168 & 29.6621 & 27.0197 & 29.8065 & 29.8986 \\
\hline 20 & 28.5606 & 26.0318 & 28.9880 & 29.0013 & 28.4521 & 26.0436 & 28.5904 & 28.6067 \\
\hline 25 & 27.5727 & 25.1740 & 27.8595 & 28.0657 & 27.5828 & 25.3848 & 27.7070 & 27.7378 \\
\hline
\end{tabular}

Table 5. The Comparing Three Different Models of MAE

\begin{tabular}{|c|c|c|c|c|c|c|c|c|}
\hline \multirow[b]{2}{*}{$\sigma^{2}$} & \multicolumn{4}{|c|}{ Peppers } & \multicolumn{4}{|c|}{ Barb } \\
\hline & $\begin{array}{c}\text { ROF } \\
\text { TV }\end{array}$ & Ying & $\mathrm{Db} 4$ & Sym4 & ROF TV & Ying & $\mathrm{Db} 4$ & Sym4 \\
\hline 10 & 3.8777 & 5.6213 & 3.8676 & 3.8038 & 4.8637 & 6.6547 & 4.9030 & 4.8760 \\
\hline 15 & 4.8590 & 6.9657 & 4.8213 & 4.7960 & 5.8950 & 8.0587 & 5.9163 & 5.8390 \\
\hline 20 & 5.7311 & 8.1453 & 5.7405 & 5.6626 & 6.8036 & 9.0224 & 6.7047 & 6.7120 \\
\hline 25 & 6.4013 & 9.1213 & 6.3854 & 6.2314 & 7.5281 & 9.9282 & 7.4997 & 7.4297 \\
\hline
\end{tabular}




\begin{tabular}{|c|c|c|c|c|c|c|c|c|}
\hline \multirow[b]{2}{*}{$\sigma^{2}$} & \multicolumn{4}{|c|}{ Brain } & \multicolumn{4}{|c|}{ Goldhill } \\
\hline & $\begin{array}{c}\text { ROF } \\
\text { TV }\end{array}$ & Ying & $\mathrm{Db} 4$ & Sym4 & ROF TV & Ying & Db4 & Sym4 \\
\hline 10 & 5.0680 & 6.6737 & 5.1141 & 5.0402 & 5.0069 & 7.1294 & 5.0311 & 4.9965 \\
\hline 15 & 6.1784 & 8.1437 & 6.0019 & 5.9826 & 6.1419 & 8.4051 & 6.2151 & 6.0777 \\
\hline 20 & 7.0583 & 9.2817 & 6.8866 & 6.7774 & 7.0413 & 9.4107 & 7.0928 & 7.0277 \\
\hline 25 & 7.8039 & 10.1688 & 7.7161 & 7.5778 & 7.7964 & 10.1797 & 7.8054 & 7.7055 \\
\hline
\end{tabular}

Previous analysis has pointed out that using gradient $|\nabla u|$ as the only description of the structure features has some disadvantages, because, on the one hand, the local structure information is not only manifested by image gradient, gradient can not precisely describe corners and textures information; on the other hand, simply using gradient to describe image direction information is not accurate in the case of noise interference. Therefore, the diffusion behavior excessively depended on gradient is hard to avoid blurring the image edges and detail features.

Lagrange multiplier $\lambda$ is a parameter related to the image gradient in TV model which can just take only one value in one diffusion process. It means that, for those images with different structures and textures, the traditional TV model lacks adaptability and may lose some details. Therefore, in this paper, the multi-resolution feature of multi-scale wavelet is introduced into the energy minimization model and a novel wavelet-domain image restoration model based on variation regularization model(WTV) is proposed. In WTV model, we make use of wavelet transform modulus to describe the image features, and then use it to construct a weighting function $\lambda_{j}$ to replace the Lagrange multiplier $\lambda$ in TV model. Experiment results show that WTV model can better describe the image structure information, reduce the adverse effect of the noise in the diffusion process and keep the details while denoising.

Figure 2 to Figure 9 show that the proposed method compare with TV model and wavelet thresholding method is more suitable for visual system and obtain a better visual effect. From the numerical comparisons of Table 4 and Table 5, we know that the proposed method denoising is significantly better than TV model and wavelet threshold method.

\section{Conclusion}

This paper analyzes the denoising principles and problems in the existing image denoising models, and emphatically probes into image denoising models based on variational partial differential equation (PDE) and wavelet transform (WT) methods. On the basis of analyzing PDE-based methods, the multi-resolution feature of multi-scale wavelet is introduced into the energy minimization model, and then a novel wavelet-domain image restoration model based on variation regularization model (WTV) is proposed. In the new WTV model, a weighting function which can describe the image structure is constructed according to the singularity of image wavelet transform modulus. Thus, the WTV model can inhibit diffusion behavior to keep the detail features in the edges and texture areas, while increase the diffusion behavior to remove noise in smooth areas. The experimental results indicate that, compared with other existing approaches, WTV model can suppress the pseudo-Gibbs effect brought by wavelet threshold shrink denoising method and overcome the cartoon effects brought by TV method, meanwhile, it can reduce the iterations and has a strong anti-noise capability.

\section{Acknowledgements}

This work was supported by the National Science Foundation of China (Grant No. 61170161) and Natural Science Foundation of Shandong Province (ZR2012FQ029). 


\section{References}

[1] P. Perona and J. Malik, "Scale-Space and Edge Detection Using Anisotropic Diffusion", IEEE Transactions on Pattern Analysis and Machine Intelligence, vol. 12, no. 7, (1990), pp. 629-639.

[2] L. Rudin, S. Osher and E. Fatemi, "Nonlinear Total Variation Based Noise Removal Algorithms", Physica D, vol. 60, no. 1-4, (1992), pp. 259-268.

[3] D. L. Donoho and I. M. Johnstone, "Ideal Spatial Adaptation Via Wavelet Shrinkage", Biometrika, vol. 81, (1994), pp. 425-455.

[4] H. Y. Zhang and Q. C. Peng, "Adaptive Image Denoising Model Based on Total Variation", Opto-Electronic Engineering, vol. 33, no. 3, (2006), pp. 50-53.

[5] T. F. Chan, J. Shen and H. M. Zhou, "Total Variation Wavelet Inpainting", Math. Image. Vision, (2006).

[6] F. Liu, "The Diffusion Filter Method of Image Based on Wavelet Transforms", China science E, vol. 6, no. 6, (2006), pp. 668-677.

[7] G. Gilboa, N. Sochen and Y. Y. Zeevi, "Texture Preserving Variational Denoising Using an Adaptive Fidelity Term”, Proc. VLSM, Nice, France, (2003).

[8] G. Gilboa, N. Sochen and Y. Y. Zeevi, "Estimation of Optimal PDE-based Denoising in The SNR Sense", CCIT Report 499, Technion, (2004).

[9] S. Bing, "Topics in Variational PDE Image Segmentation, Inpainting and Denoising", USA: University of California Los Angeles, (2003).

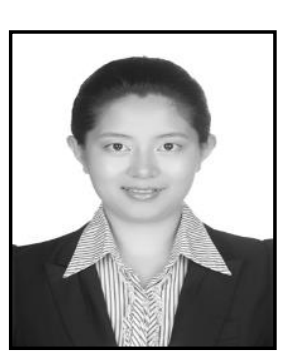

\section{Authors}

Caixia Li, she is a master-degree graduate candidate majoring in Computer Application Technology in school of information and electrical engineering of Ludong University. Her current research interests include Image Processing and Pattern Recognition.

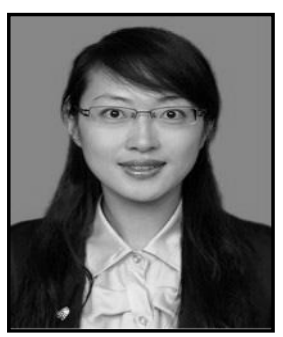

Chanjuan Liu, she received her M.Sc. in Software Engineering (2006) and PhD in Computer Application Technology (2013) from China University of Mining \& Technology (Beijing).Now she is a lecturer at school of information and electrical engineering, Ludong University. Her current research interests include different aspects of Pattern Recognition and Image Processing.

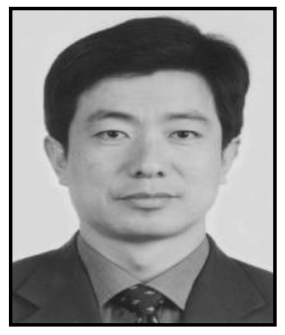

Hailin Zou, he received his $\mathrm{PhD}$ in Computer Application Technology (2002) from China University of Mining \& Technology (Beijing).Now he is full professor at school of information and electrical engineering, Ludong University. His current research interests include 3D Visualization, Pattern Recognition and Image Processing. 
International Journal of Signal Processing, Image Processing and Pattern Recognition Vol.7, No.2 (2014) 